\title{
ERRATUM for the paper: Cytology of Pericardial Effusion due to Malignancy. Kalogeraki A, Lazopoulos G, Papadakis GZ, Tamiolakis D, Karvela-Kalogeraki I, Karvelas-Kalogerakis M, Segredakis J, Papadakis M, Moustou E, Datseri G, Chalkiadakis GE, Tzardi M. Rom J Intern Med. 2016 Sep 1;54(3):179-183. doi: 10.1515/rjim-2016-0026."
}

\author{
ALEXANDRA KALOGERAKI ${ }^{1}$ \\ ${ }^{1}$ University of Crete, Medical Faculty, Greece
}

\section{Dear Editor,}

Please note that:

1. Authors listed are as follows: Kalogeraki A, Lazopoulos G, Papadakis GZ, Tamiolakis D, KarvelaKalogeraki I, Karvelas-Kalogerakis M, Segredakis J, Chalkiadakis GE. Authors that are not included any more are pathologists, they were not involved in the design and the performance of the study such as no correlation with histopathological results was made, so they wish their names to be removed.

\section{Abstract section page 179}

\section{Background.}

We remove the sentence

"Pericardial effusions with a cardiac tamponade constitute a surgical emergency and the pericardiocentesis represents the first class therapeutic recommendation".

Material and Methods.

We remove the sentence

".... and results were compared with pericardial histology results".

We correct:

“...over a $\mathbf{1 0}$ year period” as follows: “...over a $\mathbf{2 5}$ year period.”:

Results.

We remove the sentence

"There was no any false negative result in comparison with histology".

\section{Conclusions.}

Cytology provides an immediate and accurate means of diagnosis. Immunocytology is very important in the diagnostic evaluation.

\section{INTRODUCTION section page 179.}

We remove the paragraphs:

"Normal pericardium is a double-walled sac that contains the heart and the roots of the great vessels. The pericardium is composed of two different layers: an outer fibrous parietal pericardium and an inner visceral pericardium.

The inner visceral pericardium is a serous-type membrane and is located immediately outside of the myocardium. The pericardium prevents sudden dilatation of the heart, especially the right chamber, and displacement of the heart and great vessels, minimizes friction between the heart and surrounding structures, and prevents the spread of infection or cancer from the lung or pleura. The pericardium also contributes to diastolic coupling between the two ventricles. 
In between the parietal and visceral pericardium, there is a pericardial cavity filled with $10-50 \mathrm{cc}$ of fluid, an ultrafiltrate of plasma that is produced by the visceral pericardium. Pericardial fluid acts as a lubricant between the heart and the pericardium. Excess fluid or blood accumulation in this cavity is called pericardial effusion.

M-mode and 2-dimensional Doppler echocardiography is the most effective technique, and is the gold standard for the diagnosis of pericardial effusion, because it is sensitive, specific, noninvasive, and easily made available at the bedside. Pericardial effusion can be detected as an "echo-free space" on 2-dimensional echocardiography.

Small collections of pericardial fluid, which can be physiologic ( 25 to $50 \mathrm{~mL}$ ), may be visible during ventricular systole. When the amount of effusion is more than $50 \mathrm{~mL}$, an echo-free space persists throughout the cardiac cycle."

We change the sentence:

"The aim of this study is to estimate the diagnostic value of cytological examination in patients with malignant pericardial effusions in establishing the etiological diagnosis and the treatment of patients with cardiac tamponade" as follows:

"The aim of this study is to evaluate the diagnostic accuracy of cytological examination in patients with malignant pericardial effusions and suggest the type and origin of malignancy".

\section{MATERIALS AND METHODS end of page 180}

We correct:

"A total of 145 effusion samples, from 145 patients with cardiac tamponade were received from University Hospital of Crete, over a 10-year period" as follows: "A total of 145 effusion samples, from 145 patients with cardiac tamponade were received and examined in the University Hospital of Crete, over a 25year period."

We remove the sentences "Written informed consent was obtained from all participants. The pericardiocentesis was therapeutically performed in all patients."

We correct "A volume of more than $60 \mathrm{~mL}$ was submitted to cytology to ensure adequate diagnosis of pericardial fluids" as follows "A volume of more than $60 \mathrm{~mL}$ was adequate for cytologic evaluation."

\section{RESULTS page 180}

We remove the paragraphs:

"ECHOCARDIOGRAPHIC FEATURES

All the patients had a standard echocardiographic examination before Pericardiocentesis. Sixty four of patients had a large effusion (circumferential effusions with an arc width of $>1 \mathrm{~cm}$ ) on echocardiography while $34 \%$ had a moderate effusion (circumferential effusion with an arc width of $<1 \mathrm{~cm}$ at its greatest) and $2 \%$ had mild effusion (posteriorly loculated effusions of $1 \mathrm{~cm}$ or less in width). Among patients with large effusion $54.5 \%$ and $50 \%$ had documented evidence of right atrial and right ventricular collapse respectively. On 2D echocardiography $95.5 \%$ patients had circumferential and $4.5 \%$ had loculated effusion".

"Pericardial fluid was hemorrhagic in $68 \%$, serous in $14 \%$, serosanguinous in $11 \%$ and purulent in $7 \% . "$

"Of the tumor markers tested the mean concentrations of the CEA, CA 72-4 and CA 19-9 were significantly high: $(\mathrm{CEA}=350.46 \pm 1420.18 \mu \mathrm{g} / \mathrm{L}, \mathrm{CA} 19-9=1119.31 \pm 2120.37 \mathrm{kU} / \mathrm{L}, \mathrm{CA} 72-4=538.90 \pm$ $1164.33 \mathrm{kU} / \mathrm{L}) . "$

We correct as follows: "All the patients were evaluated by echocardiographic examination. In. sixty four patients a large effusion was revealed while in $34 \%$ a moderate effusion and in $2 \%$ a poor effusion was found respectively.

Pericardial fluid was hemorrhagic serous or both hemorrhagic and serous.

Of the biochemical markers measured, CEA, CA 72-4 and CA 19-9 were high.

The pericardial neoplastic samples were 145. The hematolymphoid (HL and NHL) malignancies were the most frequent cause of malignant effusion (34.5\%) (Figure 1), followed by squamous cell lung carcinoma (31.7\%), small cell lung carcinoma (22\%), lung and breast adenocarcinoma (Figure 2) (7.6\%), and malignant melanoma (4.2\%). Table 1 shows the distribution of malignancies in all effusion specimens."

We remove the sentence: "The above diagnoses were confirmed histologically." 


\section{DISCUSSION page 181}

\section{We correct the sentence:}

"The aim of our study is important for emphasizing the role of cytological examination in malignant pericardial fluids" as follows "The aim of our study is to emphasize the role of cytological examination in malignant pericardial fluids."

\section{We remove the paragraphs:}

"Small-volume pericardiocentesis specimens detect fewer malignancies and have inferior sensitivity compared with pericardial biopsy. A minimum volume of more than $60 \mathrm{~mL}$ was necessary for adequate cytologic diagnosis of malignant pericardial effusions in our settings."

"Echocardiography is the most useful diagnostic tool for evaluating patients with cardiac tamponade, and it should be performed without delay in patients if suspected.

Cardiac tamponade is a life-threatening, slow or rapid compression of the heart due to the pericardial accumulation of fluid, pus, blood, clots, or gas as a result of effusion, trauma, or rupture of the heart.

Although cardiac tamponade is considered a clinical diagnosis, clinical findings like dyspnea, hypotension, tachycardia, elevated jugular venous pressure, and pulsus paradoxus, are known to have limited sensitivity and specificity.

The most common cause of cardiac tamponade reported is malignancy, which is involved in $>50 \%$ of all tamponade cases. Especially lung cancer was involved in $>70 \%$ of cardiac tamponade of malignant origin.

When a pericardial effusion is detected by echocardiography, the next step is to assess the size of the effusion, its location, hemodynamic importance, and associated diseases."

"The diagnosis in all cases of our study required a constellation of cytology - immunocytology and correlation with the clinical history of the patient.

The cytological evaluation might be false negative in patients with lymphoma or mesothelioma, with $100 \%$ specificity but a variable sensitivity."

"However, a generally accepted system is the effusion graded as minimal (scanty), small, moderate, or large. For circumferential pericardial effusions, any pericardial effusion with less than $5 \mathrm{~mm}$ of pericardial separation in diastole (corresponding to a fluid volume of 50 to $100 \mathrm{~mL}$ ) is defined as minimal; 5 to $10 \mathrm{~mm}$ of separation as small (corresponding to a fluid volume of 100 to $250 \mathrm{~mL}$ ); 10 to $20 \mathrm{~mm}$ of separation as moderate (corresponding to a fluid volume of 250 to $500 \mathrm{~mL}$ ); and greater than $20 \mathrm{~mm}$ separation as large (corresponding to a fluid volume greater than $500 \mathrm{~mL}$. ) [16]”.

This classification may be useful in daily clinical practice. However, even the diffused and circumferential effusion dimensions of the echo-free space may be different in the views examined; therefore, it is more correct and easier to measure and annotate the dimension of the effusion and to report where it has been evaluated (e.g., $12 \mathrm{~mm}$ in the left ventricular lateral wall in the apical four-chamber view; $10 \mathrm{~mm}$ along the right atrium in the subcostal view). This methodology not only facilitates the definition of effusion size, but also allows follow-up studies by detecting changes in the amount of pericardial fluid after therapy [16].

Except for the epicardial fat, the abnormal masses attached on the epicardial surface or floating in the pericardial space must also be reported. It may be an infiltrative metastatic mass, inflammatory fibrin strands, pus, or a blood clot [16].

Other imaging modalities, such as computed tomography (CT) or magnetic resonance imaging (MRI), may be used to identify the characteristics of pericardial effusion and tamponade in the presence of a technically-limited echocardiographic study.

Currently, these modalities have adjunctive roles to echocardiography, especially in situations that show atypical hemodynamics, presence and severity of tamponade are doubtful, or when there are other unexplained conditions. For instance, in the case of pericardial effusion associated with intrathoracic malignancies, such as lung, breast, or esophageal cancer, chest CT might be useful for understanding the disease progression [16].

The definitive diagnosis of malignant pericardial effusion is established by a positive cytological examination of the pericardial fluid. However, pericardial fluid cytology, although specific, has variable sensitivity." 


\section{We correct:}

"In this study the use of immunocytology was very important, and thus we had no any false negative diagnosis" as follows: "In this study the use of immunocytology was very important."

\section{We delete:}

"Many times it was useful to perform cell blocks too"

We correct:

"Sometimes the cytology examination cannot differentiate the reactive mesothelial cells from the malignant mesothelial or metastatic cells" as follows: "Sometimes morphology alone cannot differentiate the reactive mesothelial cells from the malignant mesothelial or metastatic cells."

We delete the words in parenthesis (rhabdomyosarcomas, angiosarcomas, myxosarcomas, fibrosarcomas, leiomyosarcomas, reticulum cell sarcomas, desmoplastic small round cell tumor and liposarcomas, primary carcinomas) and correct as follows: "A primary tumor such as mesothelioma [8] a tumor arising in the pericardium(various types of sarcomas,and.primary carcinomas) [9], a mediastinal tumor (lymphoma, thymoma, seminoma or malignant teratoma) [10], a metastasized tumor or a chronic inflammatory lesion like rheumatoid or TBC pericarditis $[11,12]$ are considered in the differential diagnosis."

In a previous analysis conducted by Dragoescu and Liu [15], authors claim that "the performance of pericardial fluid cytology in detecting malignancy is better than that of pericardial biopsy, with a sensitivity of $71 \%$, and a specificity of $100 \%$, compared with $64 \%$ sensitivity and $85 \%$ specificity for the pericardial biopsy."

Echocardiography is the most effective method to detect a pericardial effusion.

We add as follows:

"Several grading systems have been developed, based on the size of the pericardial effusion [16].

Other imaging modalities, such as computed tomography (CT) or magnetic resonance imaging (MRI), are used as an adjunct.

Biochemical markers are often investigated after pericardiocentesis but their value is debated [17].

We correct:

"In conclusion, the cytological analysis of pericardial fluid has increased the certainty of the etiological diagnosis in patients with no antecedents or clinical data of neoplasia. The use of immunocytochemistry and cell blocks increases the diagnostic value of cytology" as follows: "In conclusion, the cytological analysis of pericardial fluid is helpful to establish a timely and accurate diagnosis in patients with no clinical evidence of neoplasia. The use of immunocytochemistry enhances the diagnostic performance of cytology."

We apologize for the inconvenience.

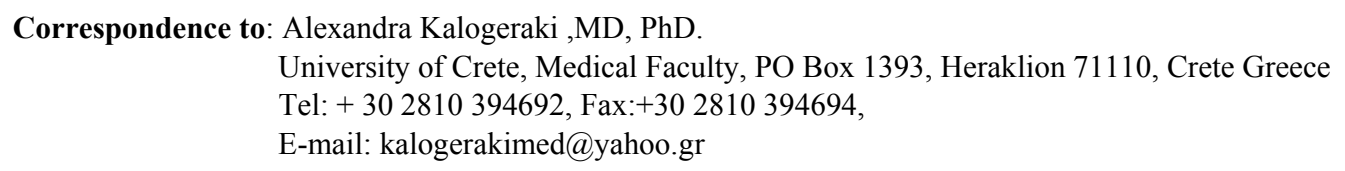

REFERENCES

1. KALOGERAKI A, LAZOPOULOS G, PAPADAKIS GZ, TAMIOLAKIS D, KARVELA-KALOGERAKI I, KARVELASKALOGERAKIS M, SEGREDAKIS J, PAPADAKIS M, MOUSTOU E, DATSERI G, CHALKIADAKIS GE, TZARDI M. Cytology of Pericardial Effusion due to Malignancy. Rom J Intern Med. 2016; 54(3):179-183. 